\title{
TWO-GENERATOR CABLE KNOTS ARE TUNNEL ONE
}

\author{
STEVEN A. BLEILER
}

(Communicated by Ronald Stern)

\begin{abstract}
A two-generator cable knot exterior is a genus two handlebody with a single two-handle attached.
\end{abstract}

Knot theorists have long studied knots via the fundamental group of the complement. These groups are finitely generated, and a naive measure of the complexity of such a group is the rank, i.e., the minimal number of generators needed to present the group. Dehn's lemma and homology show that only the unknot has a rank one (i.e., cyclic) group, so in this sense the "simpliest" nontrivial knots are those whose groups are of rank two, the so-called two-generator knots. It can be very difficult to determine the rank of a group [MKS, B]; correspondingly it can be very difficult to recognize a 2-generator knot. Indeed, in R. Kirby's famous 1978 problem list [K, 1.10], L. Moser asks if there exists a geometric characterization of this algebraic condition on knots.

A way to construct two-generator knots geometrically was given by Clark in a 1980 paper [C] and is reproduced now. Begin with a genus $g$ Heegaard splitting of the 3-sphere, expressing one of the two handlebodies as a single zerohandle with $g$ one-handles attached. Removing the zero-handle and a single one-handle produces the exterior of a knot $K$ in the 3-sphere. The cores of the remaining one-handles are said to be a set of unknotting tunnels for $K$. The cardinality of a minimal set of unknotting tunnels for a given knot $K$ is then defined to be the tunnel number of $K$ and $K$ is said to be tunnel $t$. Dually, the exterior of a tunnel $t$ knot is constructed by attaching $t$ two-handles to genus $t+1$ handelbody. Such a space deformation retracts to a wedge of $t+1$ circles with $t$ discs attached, so the group of a tunnel $t$ knot has a presentation with $t+1$ generators and $t$ relators. Thus, tunnel one knots are two-generator. Scharlemann conjectured the converse in 1984 [S], and the result here is the first step in that direction.

Received by the editors April 5, 1993.

1991 Mathematics Subject Classification. Primary 57M25; Secondary 57M05.

Key words and phrases. Two-generator knot, tunnel number, tunnel number one knot.

Supported in part by a grant from the Portland State University Research and Publications Program. 
Theorem. Two-generator cable knots are tunnel one.

Proof. The idea of the proof is to list the tunnel one and two-generator cable knots and observe that the lists agree. The torus knots are all tunnel one, so in what follows assume all cables have nontrivial companions. Also assume that the cabling is nontrivial, i.e., that the wrapping number of all cables is at least two.

The tunnel one satellite knots were classified in 1991 by Marimoto and Sakuma [MS], and per their list, the tunnel one cable knots are the $(s p q \pm 1, s)$ cables on the $(p, q)$ torus knots. It is useful to recall why (compare [E, Lemma 4.6] and [M, Theorem 1]).

For such knots one constructs a tunnel directly. Conversely, suppose that $S$, the $(r, s)$ cable on the nontrivial knot $K$, is tunnel one. Denote the manifold given by $p / q$ Dehn surgery on a knot $L$ by $L(p / q)$, and consider the reducible manifold $M=S(r s / 1)$. The manifold $M$ is the connected sum of the lens space $L(s, r)$ with the manifold $K(r / s)$. Our cable knot is tunnel one, so $M$ has Heegaard genus at most two. Heegaard genus is additive under connected sum, and the lens space $L(r, s)$ has Heegaard genus one; so the Heegaard genus of $K(r / s)$ cannot be more than one. Thus $K(r / s)$ is also a lens space with (of course) cyclic fundamental group. The cyclic surgery theorem [CGLS] coupled with the fact that $s$ is at least two then tells us that $K$ is a torus knot. Now our cable is an iterated torus knot, and the well-known classification of Dehn fillings on such knots [G, Theorem 7.5] shows that $S$ is the $(s p q \pm 1, s)$ cable on a $(p, q)$ torus knot.

To see that the $(s p q \pm 1, s)$ cables on the $(p, q)$ torus knots are precisely the two-generator cables, consider the fundamental group in place of the Heegaard genus in the argument above. Given that $S$, the $(r, s)$ cable on the nontrivial knot $K$ is a two-generator, again consider the reducible manifold $M=S(r s / 1)$. The complement of our cable has a two-generator fundamental group, and the fundamental group of $M$ is obtained from that group by adding a relator; so the fundamental group of $M$ is at most two generator. Moreover, the fundamental group of $M$ is the free product of the cyclic group $\mathbf{Z} / s \mathbf{Z}$ with the fundamental group of $K(r / s)$. A theorem of Grushko and Neumann [MKS] shows that rank is additive under the free product and the cyclic group $\mathbf{Z} / s \mathbf{Z}$ has rank one, so the rank of the fundamental group of $K(r / s)$ cannot be more than one. The fundamental group of $K(r / s)$ is thus cyclic, and once again, the cyclic surgery theorem coupled with the fact that $s$ is at least two tells us that $K$ is a torus knot. Now our cable is an iterated torus knot and we finish as before.

Two-generator knots were proven to be prime by Norwood [N1], but unfortunately his published proof [N2] appears to have a gap in the proof of Lemma 3. The theorem above has the nice corollary that "most" cables are prime non-twogenerator knots. The existence of such knots is apparently not widely known [B, p. 99] even though it was known to Crowell and Fox [CF] some thirty years ago. Denoting the $n$th elementary ideal by $E_{n}$, Crowell and Fox show that the rank of a group is bounded below by any $n$ for which $E_{n}$ is proper [CF, p. 109]. In particular, they show that the knot $9_{46}$ is prime and non-two-generator. Prime knots which cannot be shown to be non-two-generator by this method but shown to be non-two-generator by the theorem above are the $( \pm 1,2)$ cables on the Alexander polynomial 1 knots such as the Conway or Kinoshita-Terasaka knot. 


\section{REFERENCES}

[B] R. Bieri, Homological dimension of discrete groups, Queen Mary College Math. Notes, Queen Mary College, London, 1976.

[C] B. Clark, The Heegaard genus of manifolds obtained by surgery on knots and links, Internat. J. Math. Math. Sci. 3 (1980), 583-589.

[CF] R. Crowell and R. Fox, Introduction to knot theory, Graduate Texts in Math., vol. 57, Springer-Verlag, New York, 1963.

[CGLS] M. Culler, C. Gordon, J. Luecke, and P. Shalen, Dehn surgery on knots, Ann. of Math. (2) 125 (1987), 237-300.

[E] M. Eudave-Munoz, On non-simple 3-manifolds and 2-handle addition, preprint.

[G] C. Gordon, Dehn surgery on satellite knots, Trans. Amer. Math. Soc. 275 (1983), 687-708.

[K] R. Kirby, Problems in low dimensional topology, Proc. Sympos. Pure Math., vol. 32, Amer. Math. Soc., Providence, RI, 1978, pp. 273-312.

[M] Y. Moriah, A note on satellites and tunnel number, Kobe J. Math. 8 (1991), 73-79.

[MKS] W. Magus, A. Karass, and D. Solitar, Combinatorial group theory, Interscience, New York, London, and Sydney, 1966.

[MS] K. Marimoto and M. Sakuma, On unknotting tunnels for knots, Math. Ann. 289 (1991), 143-167.

[N1] F. Norwood, Every one relator knot is prime, preprint, 1979.

[N2] Every two-generator knot is prime, Proc. Amer. Math. Soc. 86 (1982), 143-147.

[S] M. Scharlemann, Tunnel number one knots satisfy the Poenaru conjecture, Topology Appl. 18 (1984), 235-258.

Department of Mathematics, Portland State University, Portland, Oregon 97207 0751

E-mail address: steve@dehn.mth.pdx.edu 